\title{
Structure in turbulent mixing layers and wakes using a chemical reaction
}

\author{
By R. BREIDENTHAL \\ Guggenheim Aeronautical Laboratory, California Institute of Technology, Pasadena
}

(Received 7 March 1980)

Plane turbulent mixing between two streams of water which contained dilute chemical reactants was studied in a new blow-down water tunnel. In a diffusion-limited reaction, a $\mathrm{pH}$ indicator, phenolphthalein, in one stream mixed and reacted with a base, sodium hydroxide, in the other stream to form a visible reaction product. The product was found to exist, as expected, in concentrated regions associated with the large, spanwise-coherent structures of the turbulence. A transition in the mixing was observed in which the aqueous mixing product increased by an order of magnitude. The transition is a consequence of the appearance and development of small-scale threedimensional motions in the flow. Downstream of the transition, the amount of mixing product was independent of Reynolds number (for an order-of-magnitude increase in the latter) and at most only weakly dependent on Schmidt number.

\section{Introduction}

Turbulent mixing layers are one of the simplest turbulent shear flows and, as such, have proved to be useful in attempts to understand the nature of turbulence. Indeed a major revision in thinking has followed the discovery and recognition of coherent large-scale structures in turbulent mixing layers (Brown \& Roshko 1974; Winant \& Browand 1974; Roshko 1976; Dimotakis \& Brown 1976). Subgrid scale modelling and other predictive schemes, which incorporate observed aspects of the large structures, are being developed. Because of the mixing layer's relative simplicity and technological significance, it is still a fruitful subject for research.

A shear layer (figure $1 a$ ) is formed when two parallel flowing streams with different speeds begin to mix downstream of the trailing edge of a splitter plate partition. When the two streams have the same speed, a wake (figures $1 b, c$ ) is formed instead. During the course of these experiments, both shear layers and wakes were studied using a splitter plate with a sharp trailing edge, as well as wakes from a thick, blunt splitter plate.

An important property of a turbulent mixing layer is its ability to mix fluid from each stream at an intimate, molecular level. While the velocity field of the flow has been studied for some time, it is only recently that the mixing has been measured. Using a small gas-sampling probe, Konrad (1977) inferred the amount of molecular scale mixing in a mixing layer between two gas streams. His results were based on the idea that, if each stream carried a dilute chemical reactant and the reaction kinetics were very fast and irreversible, then the amount of chemical product would correspond to the amount of molecular scale mixing at the reaction equivalence ratio. Such a 
(a)

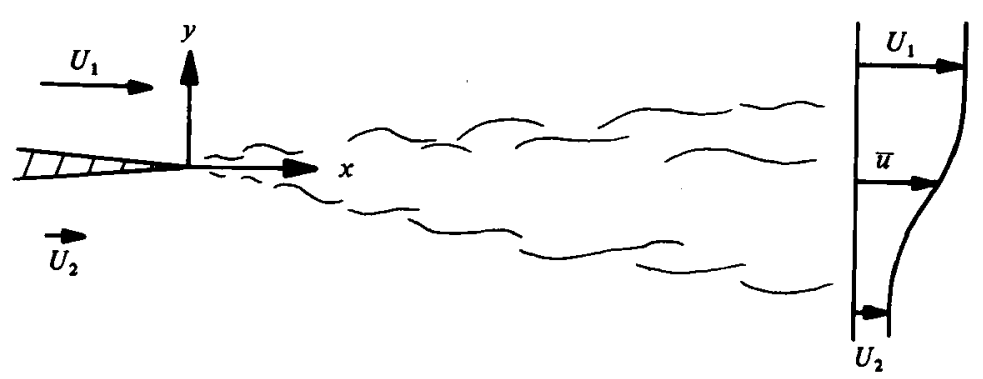

(b)

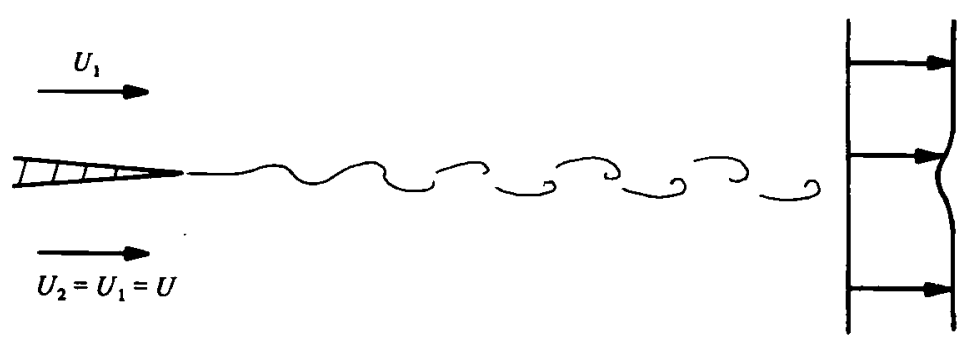

$(c)$

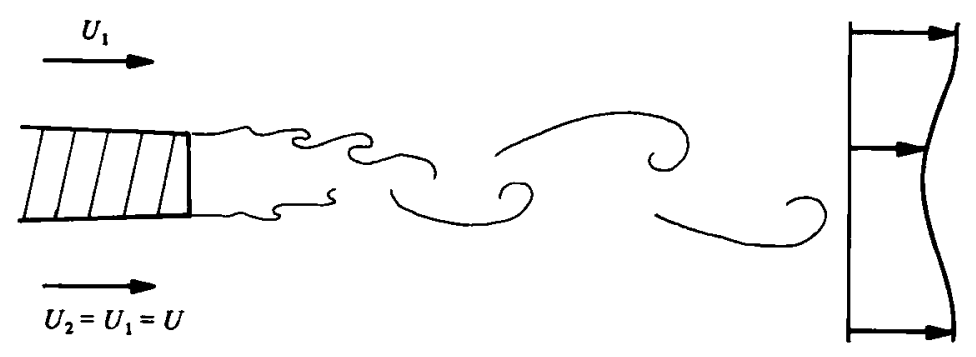

Fiqure 1. (a) Plane mixing layer. (b) Plane wake, thin trailing edge.

(c) Plane wake, thick trailing edge.

chemical system is an important limiting case of other systems, e.g. combustion with heat release or slower chemical reactions.

The ultimate step in the mixing process between two streams is molecular diffusion of one reactant toward another. A primary question that motivated the present work is 'what is the effect of the molecular diffusion rate on the mixing rate?', that is, the rate of production of product. Schmidt number $(S c \equiv v / \mathscr{D})$ is the ratio of two diffusion coefficients, that of vorticity or momentum to that of matter. It is in the nature of gases that their Schmidt numbers are almost always near unity, whereas liquids like water have Schmidt numbers three orders of magnitude larger. Thus one objective of the experiments was to explore the influence of Schmidt number (and therefore molecular diffusion) on the mixing in an aqueous shear layer $(S c \doteq 600)$ by comparing the results from Konrad's experiments in gases $(S c \doteq 0 \cdot 7)$.

The inert sampling technique of Konrad is difficult in water because of the small sampling volume required to resolve the high concentration gradients which result from the slow aqueous diffusion rate. Instead, the molecular scale mixing could be measured directly by letting a chemical reaction between the two streams produce an easily detectable reaction product. The optimum reaction would produce a 
visible reaction product which could be detected optically without intrusion into the flow.

Furthermore, the visible reaction product offers a unique flow visualization because it directly displays the mixed fluid. In contrast to an inert dye or smoke which simply diffuses away in the interesting regions of intense mixing, the visible reaction product is produced there. It is a complementary technique to conventional flow visualization and offers a potentially new view of flows which have heretofore been visualized with only conventional schemes.

Broadwell (see Witte et al. 1974) proposed a simple mixing model which predicts the effect of Schmidt number as well as Reynolds number on the mixing. The mixing process is visualized as a sequence of events which begin with the entrainment of a large blob of fresh reactant into the layer (Brown \& Roshko 1974). The blob is broken down into smaller and smaller sized lumps until the Kolmogorov microscale $\lambda_{0}$ is reached. Two time scales can be defined: a large-scale entrainment (and breakdown) time $\delta / \Delta U$ and a microscale diffusion time $\lambda_{0}^{2} / \mathscr{D}$, where $\Delta U=U_{1}-U_{2}$ is the velocity difference between the two free streams and $\delta$ is the vorticity thickness of the layer, $\Delta U /(\partial u / \partial y)_{\max }$. The ratio $T$ of these two times is $T \sim\left(\lambda_{0}^{2} / \mathscr{D}\right) /(\delta / \Delta U) \sim\left(S c / R e^{\frac{1}{2}}\right)$, where $R e \equiv \Delta U \delta / \nu \sim R e^{-3}$ from Kolmogorov's argument. When $T \gg 1$, small-scale diffusion causes the 'bottleneck' in the mixing, while, for $T \ll 1$, entrainment is the rate-limiting step. A slightly more refined argument which integrates the total time from entrainment through breakdown to the microscale yields essentially the same result. If the mixing is entrainment-limited, then the amount of product should be independent of Reynolds number and Schmidt number.

\section{Apparatus}

The design philosophy was to construct a simple, general-purpose apparatus which could be operated by one person and easily modified to vary the test-section flow conditions. A large-scale Reynolds number of at least $R e \equiv \Delta U \delta / \nu=10^{4}$ was desirable for a comparison with the high $R e$ gaseous results. For a fixed velocity ratio, $R e$ varies like the characteristic test-section dimension while the volume flow rate goes like its square. In the interest of conserving reactant chemicals and reservoir volume, a relatively small test section was chosen, $7 \times 11 \mathrm{~cm}$ span and $45 \mathrm{~cm}$ long. With a design speed of $300 \mathrm{~cm} \mathrm{~s}^{-1}$, the goal of $R e=10^{4}$ was achieved with a relatively small expenditure of reactant solution.

Since the reactants would mix and react in the test section, a closed-circuit tunnel was not feasible. Rather, a simple gravity-driven, blow-down configuration was chosen, with the reactant solutions prepared in batches before a run.

A schematic sketch of the apparatus is shown in figure 2. Each reservoir has a capacity of about $10^{3}$ l, permitting a $30 \mathrm{~s}$ run at the design speed of $300 \mathrm{~cm} \mathrm{~s}^{-1}$. Owing to the relatively small change in water level, the flow speed changes by less than $2 \%$ in $10 \mathrm{~s}$. Steady-state flow is typically established in 2-4 s. The free-stream turbulence intensity $\left(u^{\prime 2} / U^{2}\right)^{\frac{1}{2}}$ was measured by a laser-Doppler velocimeter $\dagger$ to be slightly less than $0.5 \%$ at the beginning of the test section for several tunnel speeds spanning the velocity range studied.

† With the help of Mr Manooch Mohseni-Koochesfahani and Dr Keith Koenig. 


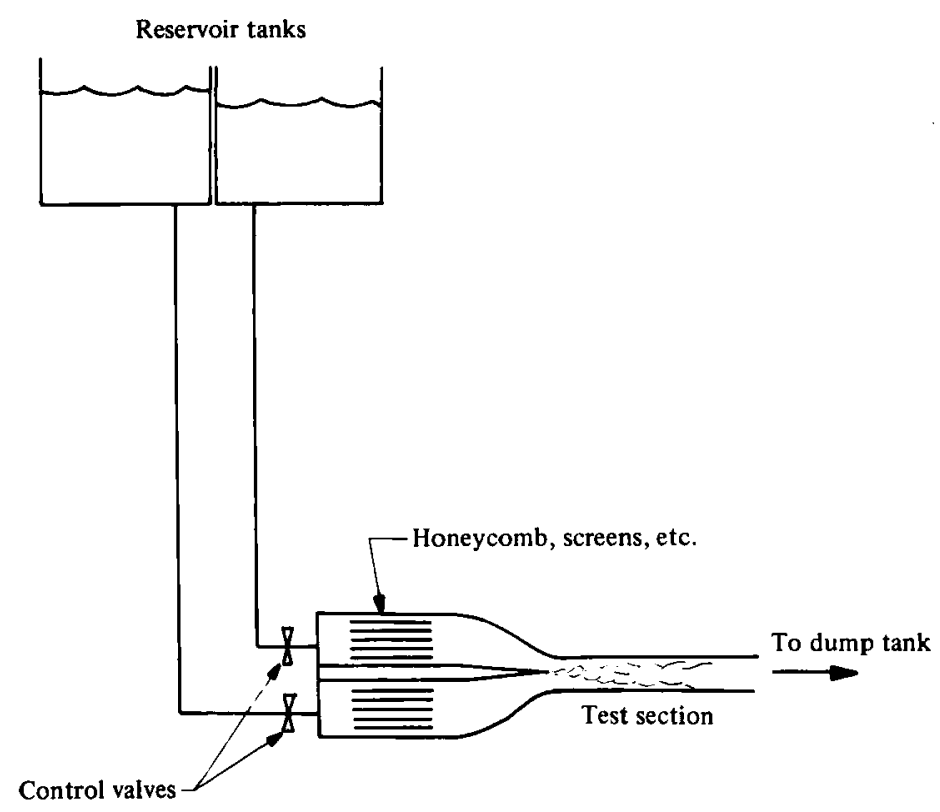

Figure 2. Sketch of the apparatus.

The nozzle contour is defined by tangent circular arcs, 48 and $11 \mathrm{~cm}$ radius, with the latter tangent to the test section. The 'thin' splitter plate is a wedge of $8^{\circ}$ total angle whose trailing-edge thickness is less than $0.003 \mathrm{~cm}$. A 'thick' splitter plate is a truncated wedge of the same length whose trailing-edge thickness is $0.635 \mathrm{~cm}$.

A stainless-steel traversing mechanism, driven by a digitally controlled stepping motor, can move a Pitot tube probe in increments of $0.0066 \mathrm{~cm}$ across the test section to measure mean velocity profiles.

\section{Measuring techniques}

\subsection{Passive scalar technique}

The amount of molecular-scale mixing in a turbulent shear layer between two streams could be measured in at least two ways. Perhaps the most obvious approach is the passive scalar contaminant technique illustrated in figure 3. An inert scalar, for example a blue dye, is introduced into one reservoir. A diffusion interface always exists within the mixing layer which separates the blue fluid from the clear, undyed stream. Within the interface the blue dye diffuses into the clear fluid; all the molecularscale mixing occurs within the interface.

Using this technique the amount of blue dye within a small sampling volume would be measured as a function of time. If the sampling volume were small enough to measure the true, local concentration of the inert dye, it would be possible, using 'Toor's analysis (Toor 1962) to infer the amount of chemical product that would be formed if each stream contained dilute reactants which rapidly react in an irreversible reaction. On the other hand, if the sampling volume is not small compared to the smallest concentration scales, i.e. the interface thickness, then the probe will smooth out the actual concentration fluctuations and thus overestimate the amount of product formed. The inert technique always yields an upper bound on the actual molecular-scale mixing 


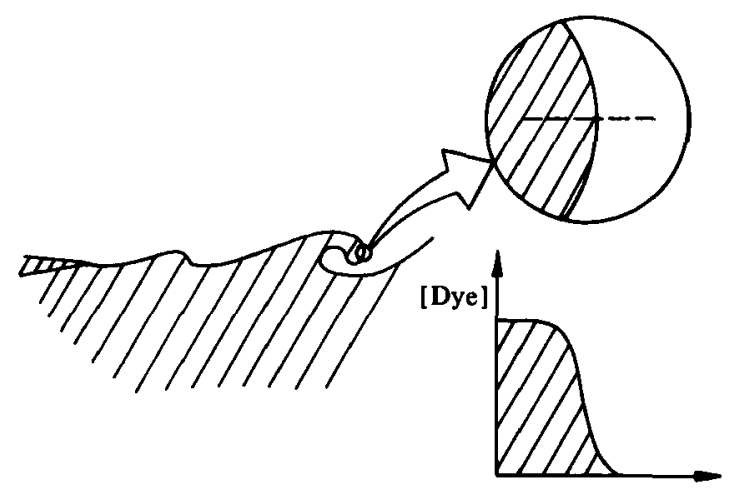

Figure 3. Inert scalar technique.

(in contrast to the reaction technique, described below, which gives a lower bound).

The diffusion interface can become quite thin in a high-Schmidt-number fluid like water. For typical high-Reynolds-number conditions in the present apparatus, the interface is estimated to be as thin as the wavelength of light (thickness $\sim\left(\mathscr{D} / \sigma_{0}\right)^{\frac{1}{2}}$, where $\sigma_{0} \sim(\Delta U / \delta) R e^{\frac{1}{2}}$ is the Kolmogorov scale strain rate). If so, it is difficult to construct even an optical sampling volume with dimensions small compared to the interface thickness. The interface could be several orders of magnitude thicker and still present a difficult problem experimentally. Of course, one could achieve a highReynolds-number flow by building a very large apparatus with flow speed so low that the diffusion interface would be thick compared to an obtainable probe size. This approach, however, was not practical.

\subsection{Chemical reaction technique}

The problem of finite probe size would be solved if a process could be found which would measure the mixing at the molecular level and macroscopically display the correct answer independent of the interface thickness. Such a process is a second-order chemical reaction: $A+B \longrightarrow C$. If dilute reactant $A$ is added to stream one, and $B$ to stream two; and if they rapidly react in an irreversible manner when mixed at the molecular level to form a reaction product $C$ (an 'ideal' reaction), then the amount of product formed is just equal to the amount of molecular-scale mixing between the two streams at the reaction equivalence ratio. Further, if the reaction product happens to be visible, then the amount of mixing can be measured optically, in a non-obtrusive way.

In contrast to the passive case, the finite size of the sampling volume in the reacting flow does not inherently overestimate the amount of mixing (see figure 4). If the reaction is 'ideal', then the total amount of mixed fluid at the reaction equivalence ratio within the sampling volume is just equal to the total amount of product there, no matter how big the sampling volume, or how thin the diffusion interface. Integrating is experimentally easier than differentiating. This attractive characteristic permits accurate aqueous mixing measurements at fairly high Reynolds numbers in a reasonably sized laboratory apparatus.

An ideal, irreversible reaction was not found; instead, it was approximated by a reversible one. (Thus the mixing inferred was a lower bound.) Phenolphthalein, a 


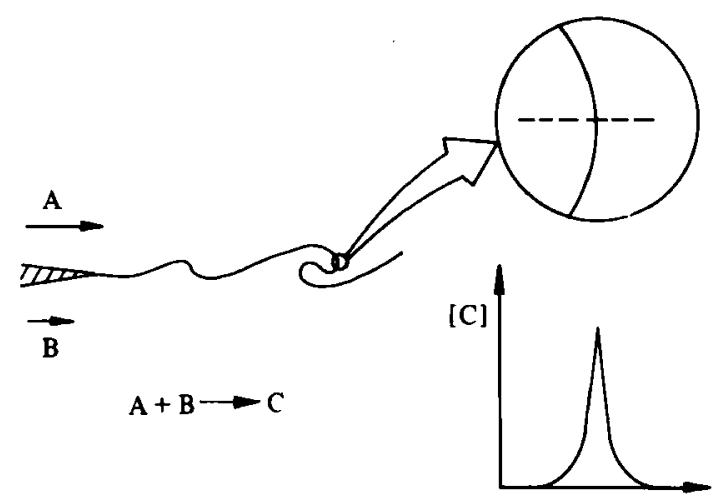

Figure 4. Reacting technique.

common $\mathrm{pH}$ indicator, reacts with a base, sodium hydroxide, in a complex series of steps (Kolthoff 1937). The overall reaction is

$$
\text { phenolphthalein }+2(\mathrm{OH})^{-} \longrightarrow \text { red product. }
$$

The reaction, while not ideal, was chosen for several reasons. It is believed to be diffusion-limited in the chemical sense and is thus very fast (Czerlinski \& Eigen 1959; Caldin 1964). The reactants are more or less water soluble, non-toxic, inexpensive and transparent. The red product is strongly visible so that dilute concentrations are sufficient for detection. Dilute reactants are unobtrusive so that the turbulence is unaffected by the chemistry, i.e. there is little surface tension, buoyancy, heat release, etc.

The experiments were performed at an equivalence ratio of about $10^{2}$, with an excess of $(\mathrm{OH})^{-}$. This was dictated by the $\mathrm{pH}$ transition range of phenolphthalein and its limited solubility in water. The free-stream phenolphthalein concentration was about $10^{-5} \mathrm{M}$ and the $\mathrm{pH}$ of the alkaline solution was about $11 \cdot 7$. This value was chosen to minimize reversibility effects and the disappearance of red product due to another reaction at very high $\mathrm{pH}$ levels (see appendix).

The amount of red product was measured by the attenuation of a narrow beam of green light (see figure 5) which passed through the layer, oriented normal to the plane of the layer. The beam width was defined by two apertures, ranging in diameter from 150 to $2000 \mu \mathrm{m}$. A narrow-band optical filter (35 \& half-maximum bandwidth) selected the $5461 \AA$ green mercury line of the light source, which is near the phenolphthalein red product absorption maximum of $5530 \AA$.

An effective product thickness $P$ was defined in a manner similar to a boundarylayer displacement thickness

$$
P \equiv \int_{-\infty}^{\infty} \frac{[\mathrm{C}](x, y, z, t)}{[\mathrm{B}]_{\infty}} d y .
$$

The integral of the product concentration along the light beam, normalized by the free-stream concentration of reactant $B$ (phenolphthalein) is the equivalent thickness of the amount of product in the layer, and can be compared to the vorticity thickness $\delta$. The ratio $P / \delta$ is, roughly speaking, the fraction of the layer filled with mixed fluid. 


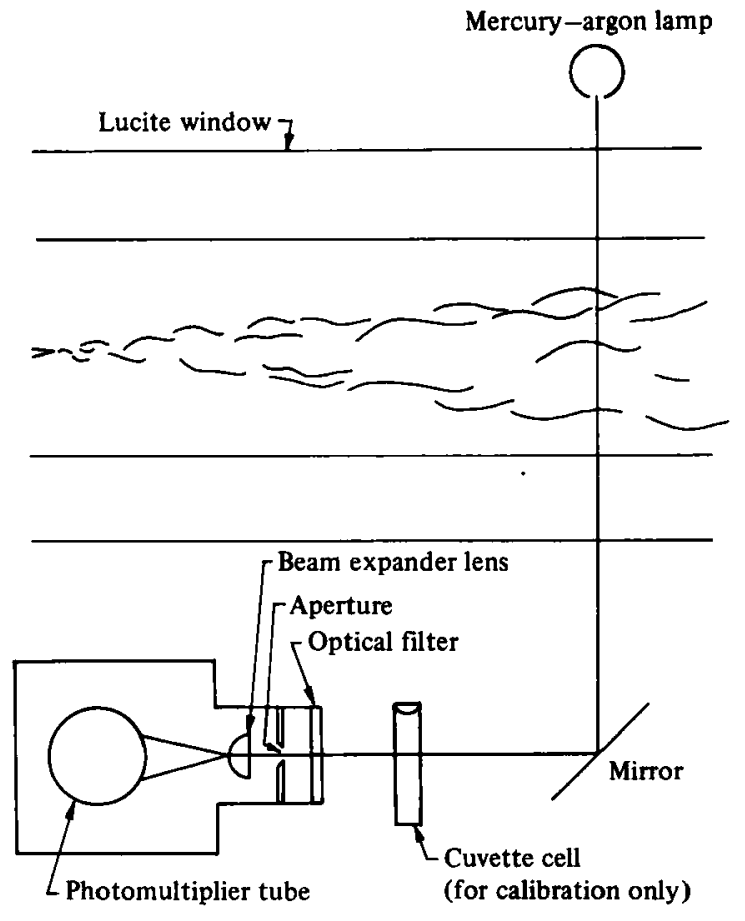

Figure 5. Optical system.

\section{Flow visualization}

As mentioned in the introduction a very useful advantage of the visible reaction product is the unique flow visualization it affords. The slow diffusion rates of vorticity and matter in water are well-known advantages in conventional flow visualization, permitting lower flow speeds for a given Reynolds number and higher-resolution photographs.

Perhaps the most intriguing feature of reacting flow visualization is the relationship between vorticity and chemical product. Initially, the vorticity and product are both concentrated in thin sheets at the splitter plate trailing edge. Both convect with the flow and diffuse down their concentration gradients (at different rates if $S c \neq 1$ ). In a two-dimensional, constant-density flow vorticity is not stretched and behaves just like a scalar contaminant, satisfying the Eulerian heat equation

$$
\frac{D \omega}{D t}=\nu \nabla^{2} \omega
$$

The local vorticity thickness, $\delta_{\text {local }}$, for an isolated sheet in two-dimensional flow is just proportional to the local product thickness $P$ times $S c^{\frac{1}{2}}$, since both behave like scalar contaminants diffusing at different rates. The actual instantaneous distributions of vorticity and product within the sheet are not identical (figure 6), since product is continually being produced at the reaction surface, whereas vorticity is not. (An inert dye would, however, label the vorticity provided $S c=1$.) Thus, within the sheet, vorticity is not precisely labelled by product, even for Schmidt number unity and two-dimensional flow.

As long as the sheet is isolated, i.e. its thickness is small compared to both its radius 


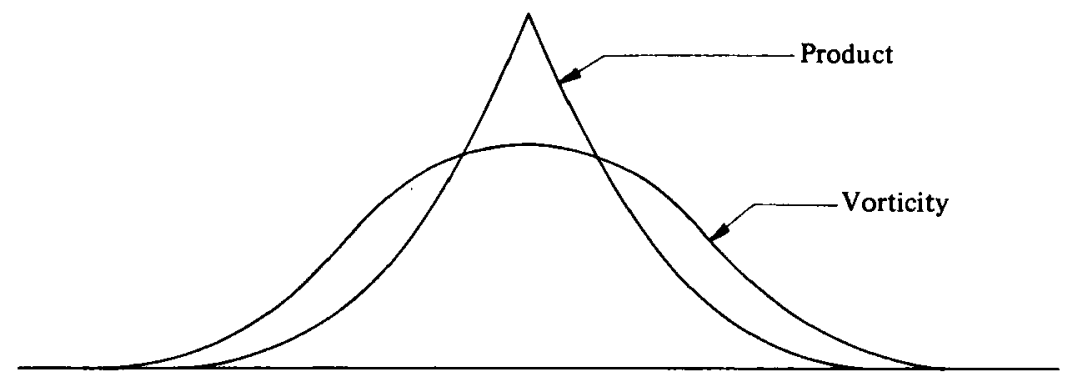

Figure 6. Product and vorticity distributions within the interface.

of curvature and the distance to any other near-by sheets, the maximum vorticity surface near the centre of the sheet will approximately coincide with the reaction surface, the surface of maximum product. In this global sense, the product labels the vorticity for an isolated sheet, regardless of Schmidt number.

If the sheet is not isolated, 'flame shortening' (Marble \& Broadwell 1977) and the analogous 'vortex sheet shortening' effects can alter the ratio $(P / \delta)_{10 c a l}$. And, if the flow is three-dimensional, vortex stretching will amplify and rotate the vorticity vector. The ohemical product is not amplified by stretching, of course, since it is a scalar.

In spite of the fact that the product does not precisely label the vorticity because of flame-shortening and vorticity-stretching effects, it may nonetheless approximately label it globally. Stretching only can amplify vorticity where vorticity already exists. One might argue that for $S c \gg 1$ vorticity can diffuse into an irrotational region faster than the product and then be amplified there by stretehing to a very large absolute value. While such events must be common, if the Reynolds number is reasonably large the vorticity cannot diffuse very far compared with the large scales in the turbulence. The viscous length is small and convection rather than diffusion dominates the transport of vorticity. From a global view, then, the chemical product may approximately label the vorticity.

\section{1.}

(a) Shear-layer flow pictures. A side view of the reacting shear layer is shown in figure $7(a)$. The lower stream is coloured with an inert blue dye which appears as light grey in the monochrome photograph. The upper, high-speed stream is transparent so that the entrainment of the blue and clear fluids into the layer is visible. The reaction product is red (dark grey in monochrome) and resides in regions associated with the large vortical structures. The photograph strongly implies that these vortices dominate both the mixing and the distribution of mixed fluid, at least for these flow conditions.

Figure 8 is a plan view of the flow in the transition region. Several features of the photograph are significant. The basic two-dimensionality of the instantaneous product distribution is evident. The flow near the side walls is somewhat disturbed. When this was first observed, it was thought to be an inherent feature of the shear-layer interaction with the wall. However, it may also be due to a secondary flow which is produced in the corners of the test section by the upstream nozzle contraction, resulting in a perturbed wall region. In any event, the flow near the test section centre-line appears reasonably clean, and all measurements were made there. 


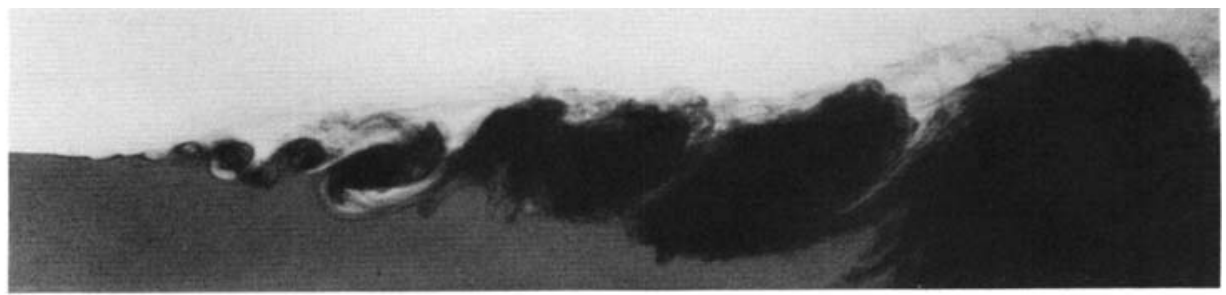

(a)

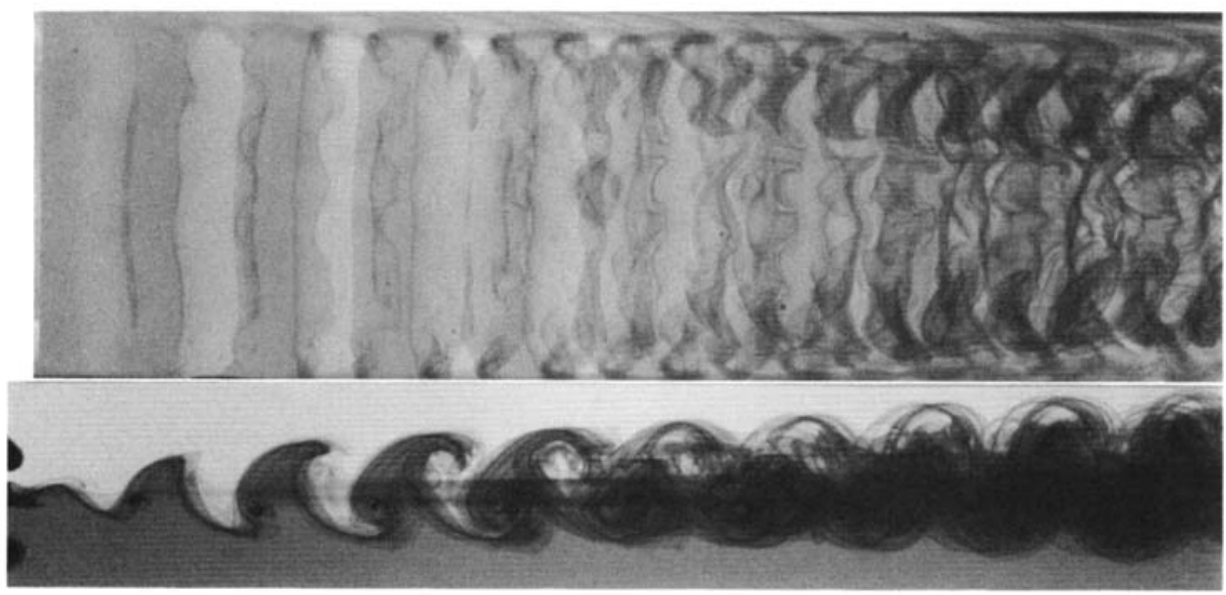

(b)

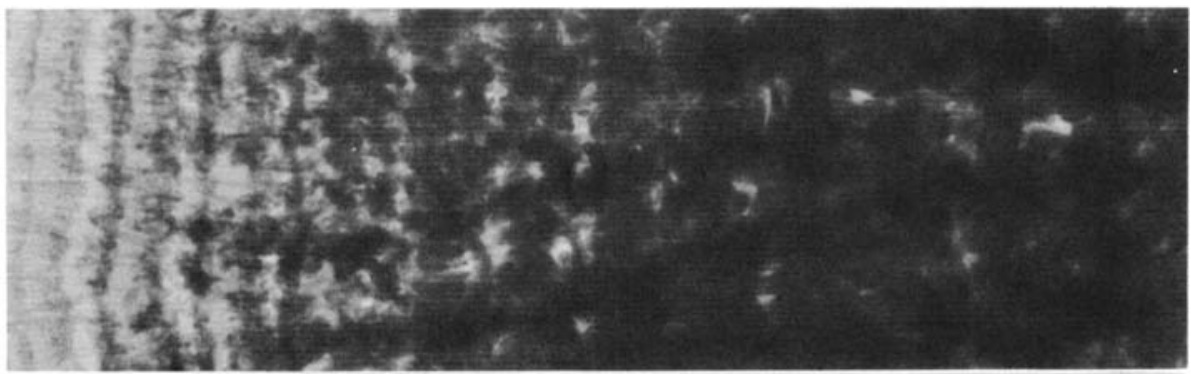

(c)

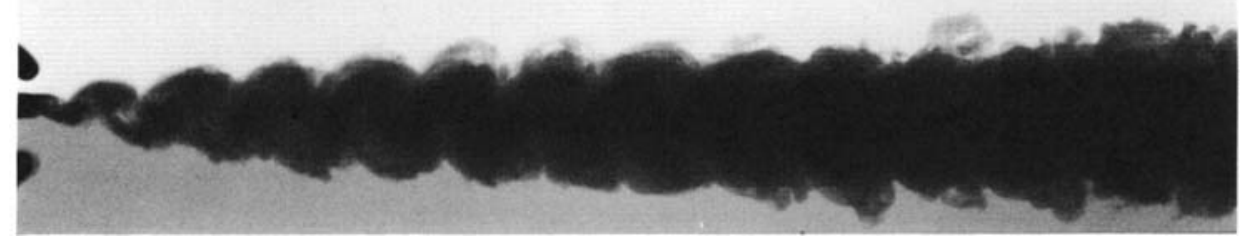

Figure 7. Reacting shear layer and thick wake. (a) $U_{1}=37 \mathrm{~cm} \mathrm{~s}^{-1}$ (top stream), $r=0 \cdot 36$. (b) $U d / \nu=200$. (c) $U d / \nu=1.6 \times 10^{4}$.

(b) The wiggle disturbance. The most interesting feature of figure 8 is the spanwisesinuous disturbance. Upstream of the 'wiggle' the flow is two-dimensional. Streamwise lines are visible downstream of the wiggle and the flow is clearly three-dimensional there, although the large-scale structures maintain their spanwise coherence.

Similar streamwise streaks have been observed by shadowgraph photography in 


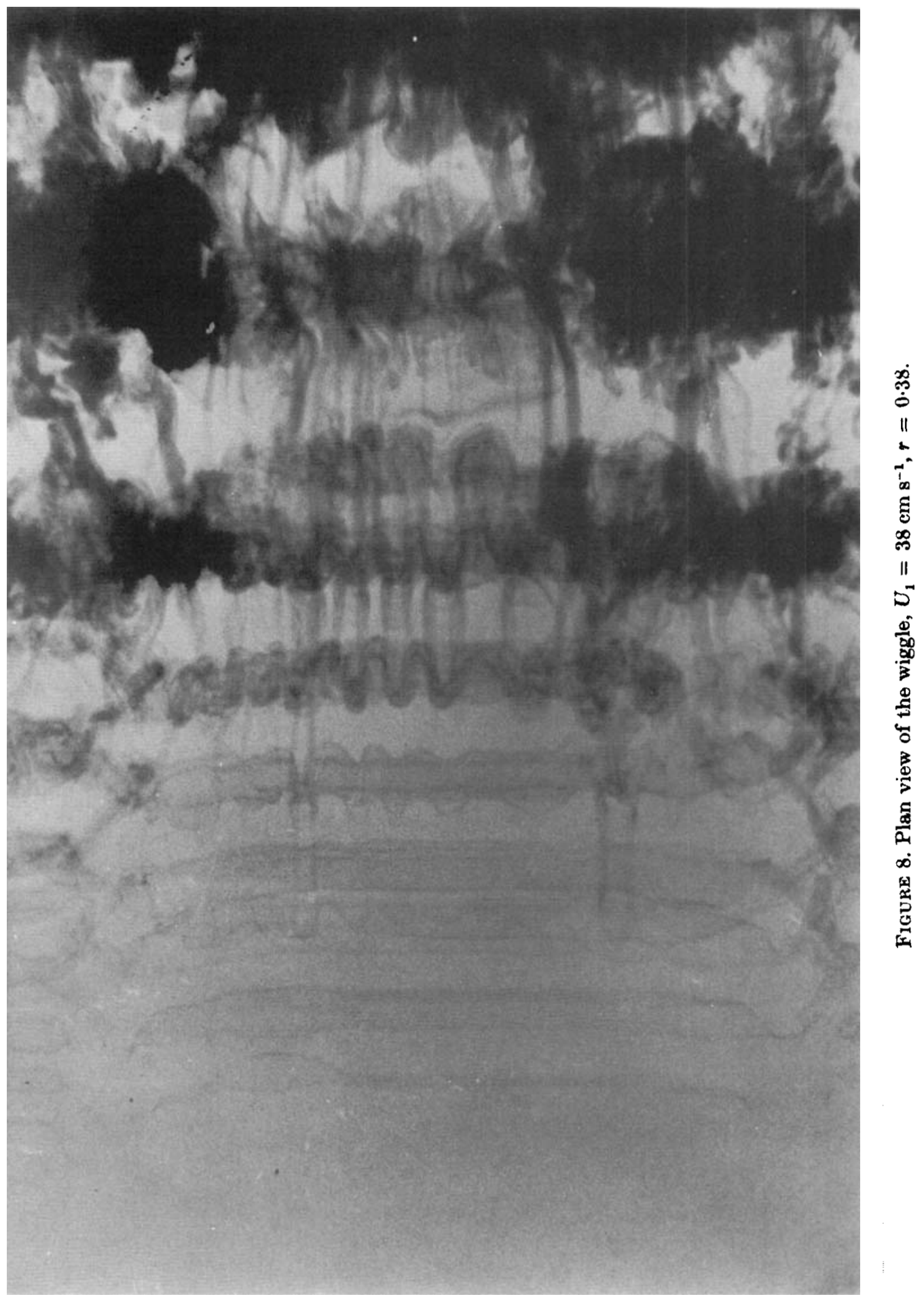


the Brown-Roshko gas apparatus for some time (Konrad 1977). They have been interpreted as streamwise vortices of alternating sign. Their origin was, however, obscure. In figure 8 another wiggle is apparently evolving in the neighbouring vortex upstream of the wiggle. The outer sheet of the vortex is corrugated, while the inner sheet appears relatively unperturbed. It is tempting to conclude that this is characteristic of the wiggle formation. The average wavelength of the sinuous wiggle was measured from photographs to be about 1.1 times the wavelength of the initial twodimensional Kelvin-Helmholtz waves. After the wiggle is formed, it is stretched by the global strain field of the flow. Its amplitude grows rapidly, while its wavelength remains constant. There are indications of this process in figure 8 . As discussed by Bernal et al. (1979) and Breidenthal (1979), the wiggle is not always nor at all conditions the only way in which three-dimensionality is introduced into the flow.

A time exposure of the plan view is shown in figure 9. The streamwise streaks represent long-time-averaged spanwise variations in the mixing. The streaks first appear at the onset of the wiggle and extend to the end of the test section. The relative amplitude of the spanwise variations appears to be maximum a short distance downstream of the wiggle location.

A photograph of the plan view at large Reynolds number (figure 10) demonstrates that, despite the presence of strong three-dimensional, small-scale motions in the flow, the large structure is basically two-dimensional, as indicated by the product distribution.

\subsection{Wake flow pictures}

The thick wake is shown in figure $7(b, c)$ (plate 1). At low values of $R e_{w} \equiv U_{\infty} d / v$ the thick wake is laminar and forms a plane interface downstream of the base flow closure region. As the Reynolds number increases, the familiar wake behaviour of quasi-twodimensional vortex shedding is apparent. Three-dimensional motions appear at $R e_{w}=150$ to 200 . Further increase in $R e_{w}$ results in a highly three-dimensional, smallscale field superimposed on the quasi-two-dimensional large-scale vortex street.

\section{Product measurements}

\subsection{Shear-layer time histories}

As discussed in §3.2, the amount of product was measured by passing a narrow beam of light through the layer normal to its plane. The red product attenuates the beam, allowing the instantaneous amount of product along the light path to be calculated and recorded as a function of time. Time histories of the equivalence product thickness $P$ (equation 3.2) are shown for two Reynolds numbers in figure 11. The shear layer has about the same thickness in both runs, yet the average value of $P$ (indicated on the vertical axis) is much higher at the larger Reynolds number. The cause of this mixing transition is the presence of small-scale three-dimensional motions at large $R e$. There are impressively large fluctuations in the instantaneous value of $P$ at the higher Reynolds number, the large excursions corresponding to large vortex structures convecting through the beam. The average time interval for the passage of the large structures is indicated, where $l$ is the mean vortex spacing.

Figure 12 is a similar time history of $P$ above the transition but with a compressed time scale. Flectronic noise accounts for occasional excursions of the signal below 


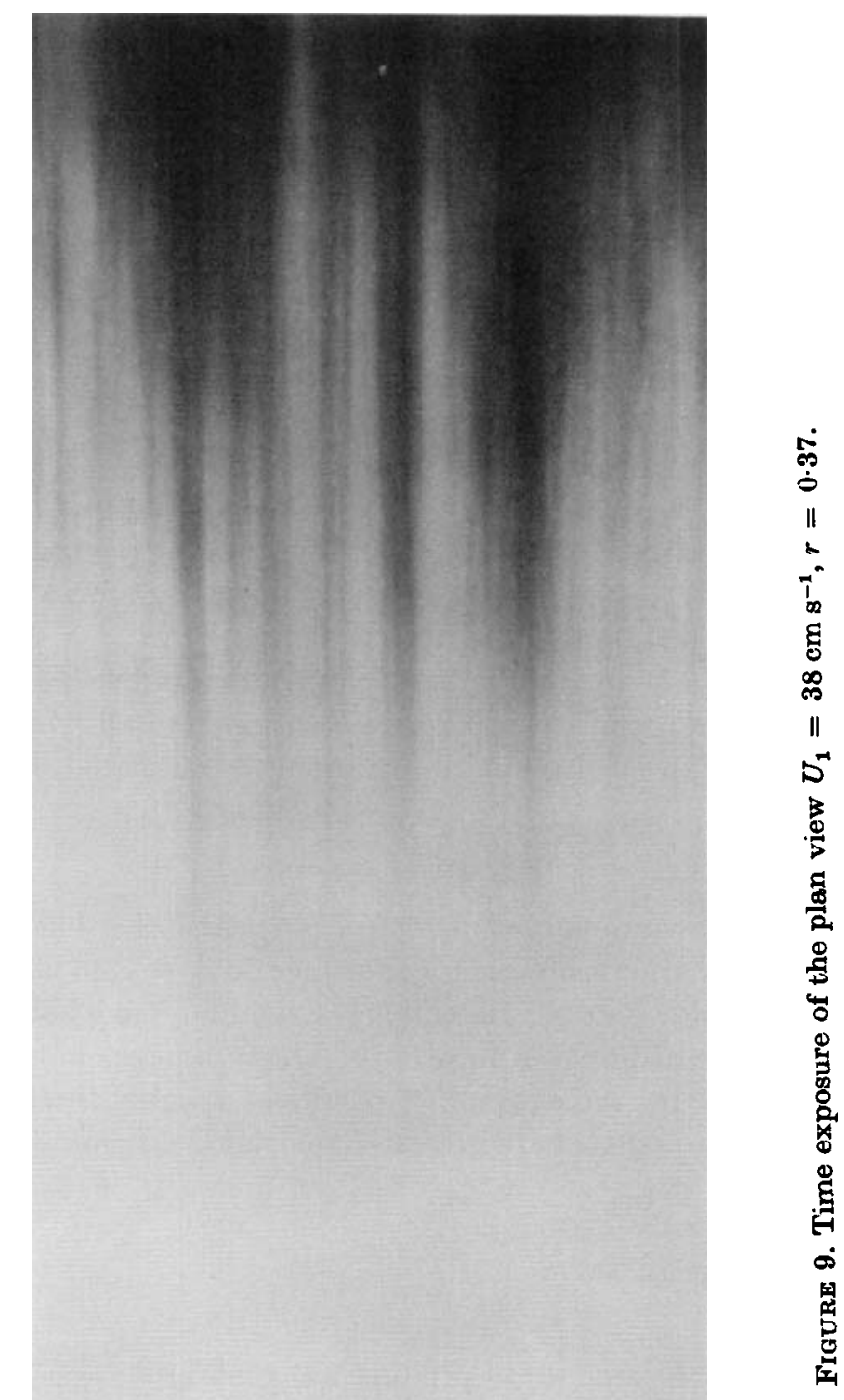




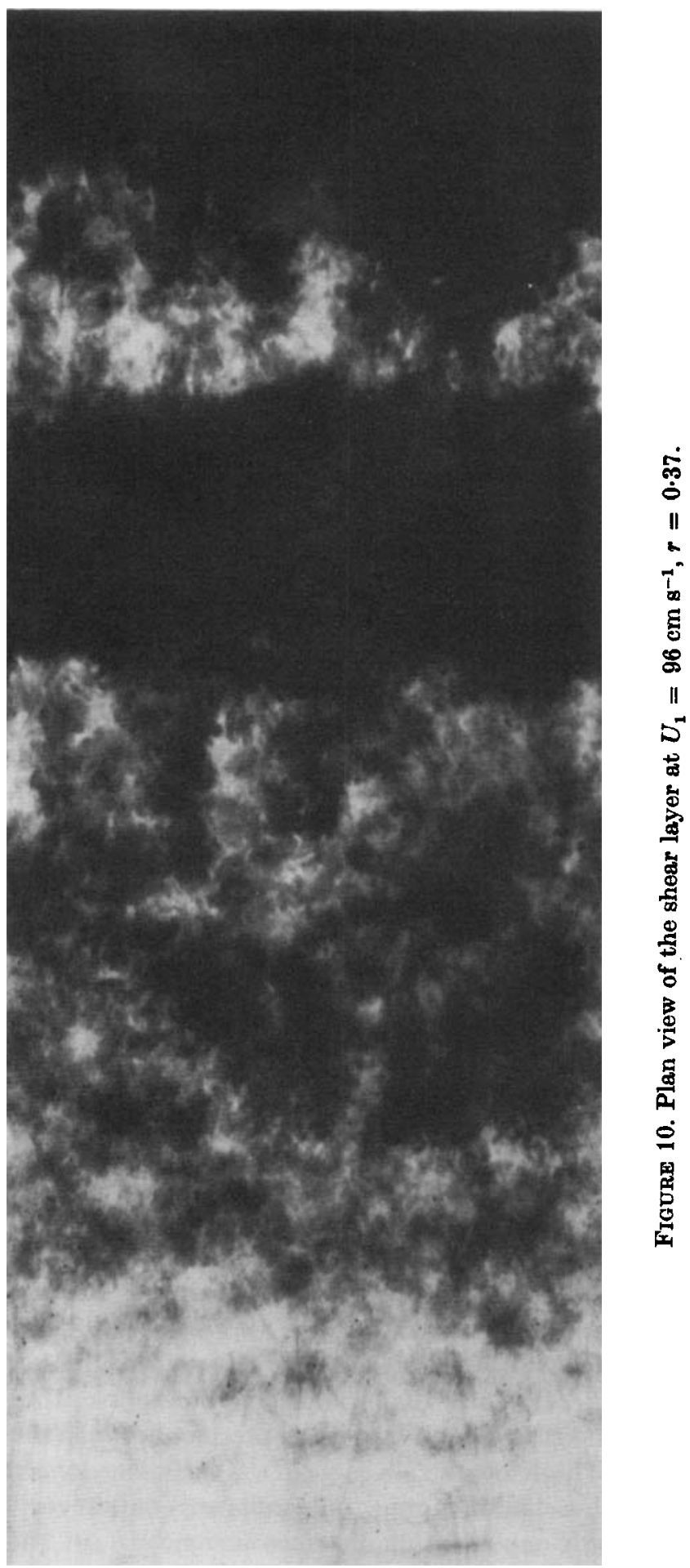




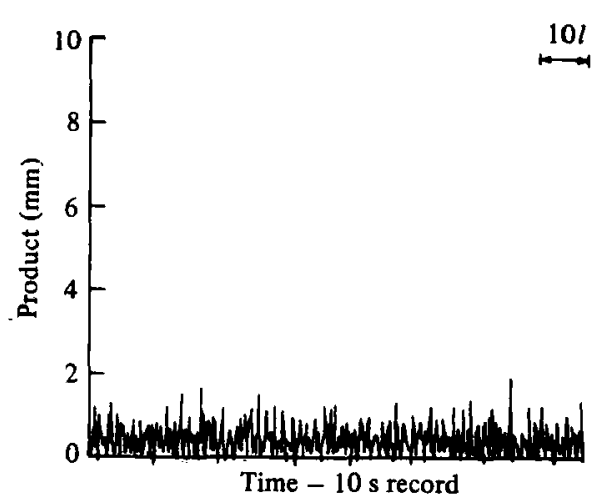

(a)

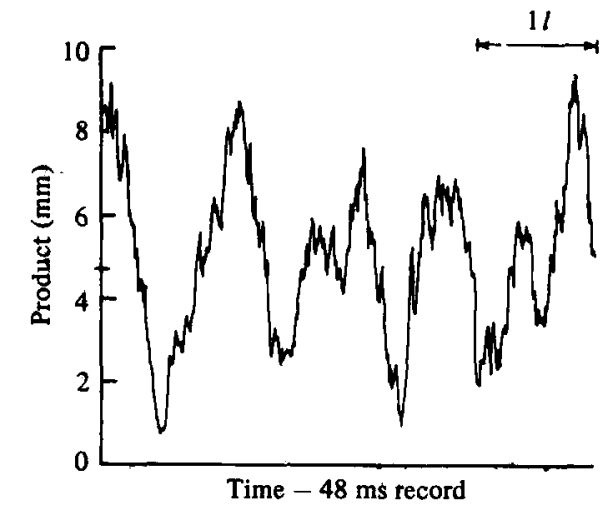

(b)

Fraure 11. Time histories of the product thickness for the shear layer. (a) $U_{1}=35 \mathrm{~cm} \mathrm{~s}^{-1}$, $r=0.36, x=14 \mathrm{~cm}$. (b) $U_{1}=257 \mathrm{~cm} \mathrm{~s}^{-1}, r=0.35, x=15 \mathrm{~cm}$.

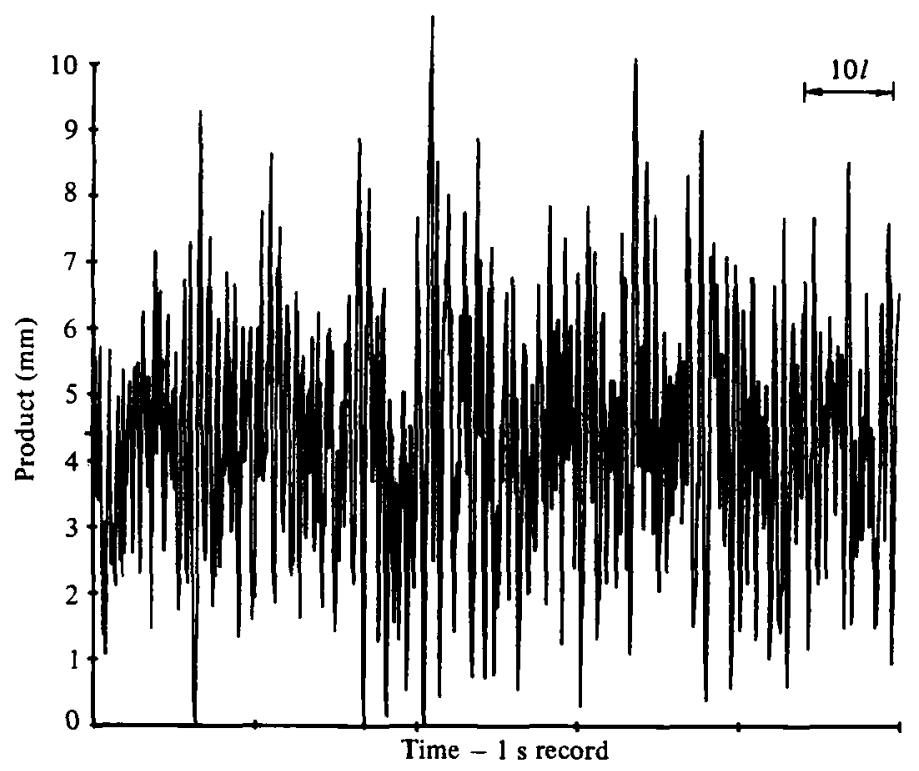

Fraure 12. Time history with a compressed time scale. $U_{1}=465 \mathrm{~cm} \mathrm{~s}^{-1}, r=0.37, x=14 \mathrm{~cm}$.

the axis $P=0$. Many large structures have passed through the beam during this long record. At several sampling times during the record the light beam detected almost no product, indicating that it is occasionally possible to see almost entirely through the layer even at large $R e$, cf. figure 10. Topologically, of course, there must always be a reaction interface and thus a finite amount of product between the two reactant fluids. However, because of high local strain rates in the turbulence this interface may locally be very thin in a high-Schmidt-number fluid and thus contain very little product. If the light beam pierces only one such thin interface at some instant, then the instantaneous product thickness is very small. 


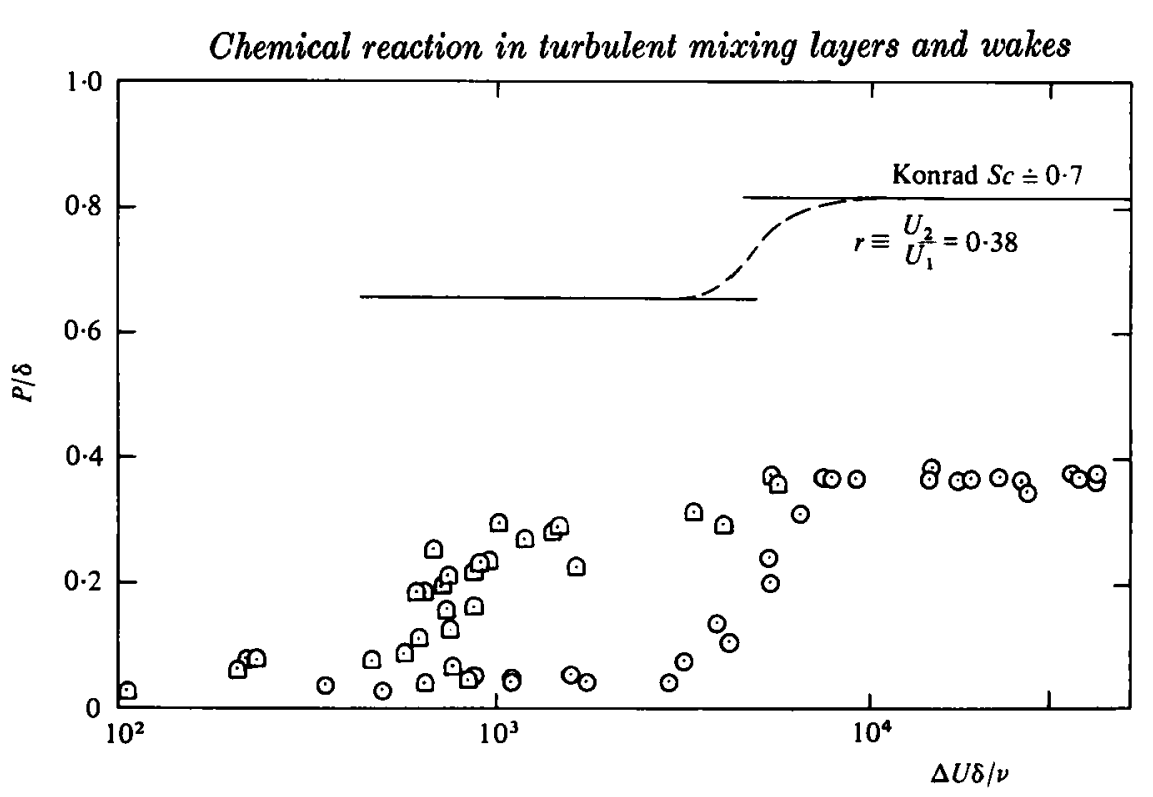

Figure 13. Effects of large-scale Reynolds number, Schimdt number and velocity ratio on the mixing. $\square, r=U_{2} / U_{1} \doteq 0 \cdot 76, S c \doteq 600 ; \odot, r=0.38, S c \doteq 600$.

\subsection{Effect of Schmidt number}

The main results of the mixing measurements for a velocity ratio of $r=0.38$ are shown in figure 13 and have been briefly reported previously (Breidenthal 1979). The (mean) equivalent product thickness $P$ normalized by the vorticity thickness $\delta$ is plotted as a function of large-scale Reynolds number $R e=\Delta U \delta / \nu$. The full-line curve is inferred from Konrad's (1977) results for a shear layer between two different gases. The highest equivalence ratio $f$ that Konrad discussed was 10, while the phenolphthalein chemistry constrains the present work to an equivalence ratio of a few hundred. Fortunately, Konrad found that the mixing in his experiment appears to approach its asymptotic value at an equivalence ratio of 10 , and little change is expected for larger values of $f$. The physical explanation is that for a large enough equivalence ratio the amount of product formed is just limited by the entrainment of the scarce reactant into the layer, since there is an abundance of excess reactant there. Thus in figure 14 the present work at $f \approx 200$ can be compared with Konrad's results for $f=10$.

The most obvious feature is the rapid increase in $P / \delta$ at $R e=5000$. At the transition $P / \delta$ jumps about $25 \%$ in the gaseous flow and about a factor of 7 in the aqueous layer.

Above the transition the amount of mixing is seen to be independent of Reynolds number and only a weak function of Schmidt number. The Schmidt number differs in the two cases by three orders of magnitude, yet $P / \delta$ changes by about a factor of 2 or less. Konrad employed the inert scalar contaminant technique which, as discussed in \$3.1, always yields an upper bound on the actual mixing. In the present study, the chemistry is reversible and the light beam is of finite width, so that the aqueous measurements are a lower bound on the mixing. Since the gaseous and aqueous measurements are upper and lower bounds, respectively, on the mixing, it is possible that the actual difference in mixing at high Reynolds number is considerably less than indicated.

Below the transition, the mixing is a strong function of Sohmidt number. The gas and water measurements differ by about a factor of 12 here. If there are no flame- 


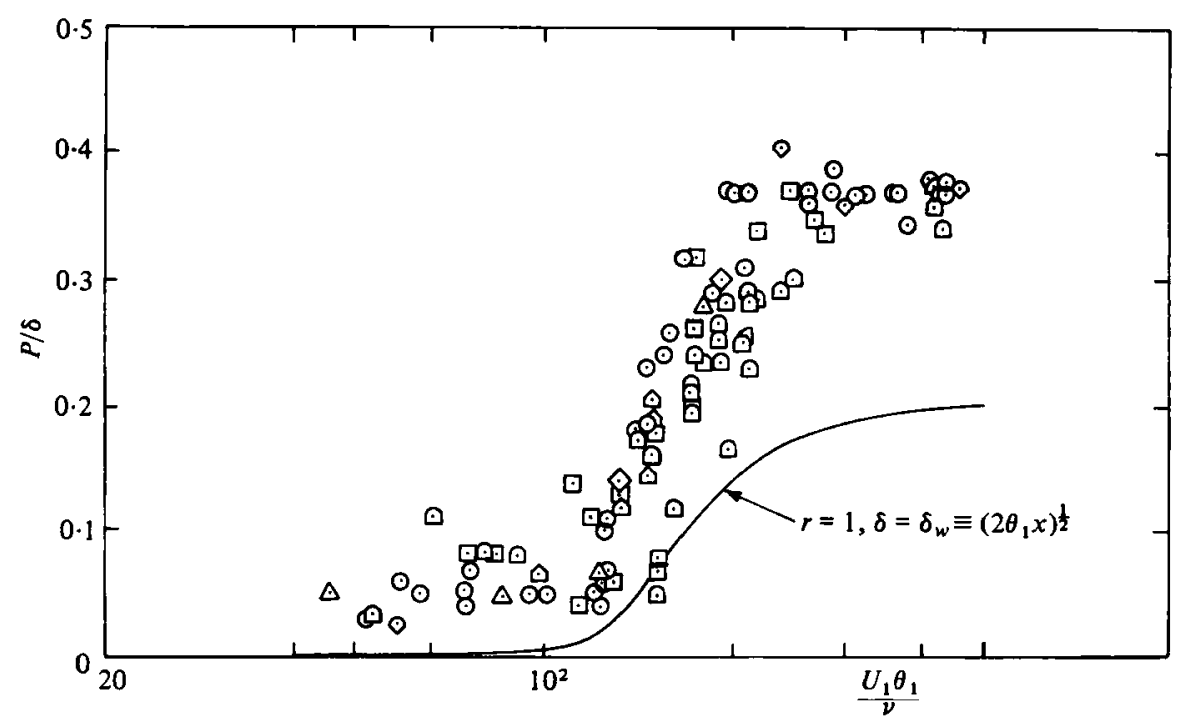

FIGURE 14. Effect of initial Reynolds number on the shear-layer transition. Values of $r\left(=U_{2} / U_{1}\right)$ : $\odot, 0.38 ; \triangle, 0.45 ; \nabla, 0.50 ; \odot, 0.57 ; \square, 0.62 ; \odot 0.67 ; \triangle, 0.76 ; \odot, 0.80$.

shortening effects, the mixing should vary like $S c^{-\frac{1}{2}}$ even though the vortex sheet is convoluted. The observed mixing difference in the two fluids is somewhat less than this; therefore there may be flame shortening in the gaseous flow. The effect should not be too surprising. Since $S c \sim 1$ for the gas the local product and vorticity sheets are about the same thickness, ignoring the initial boundary layers. (As the flow is initially two-dimensional, no appreciable vortex amplification by stretching can occur.) If the vortex-sheet radius of curvature at the vortex core is about equal to the sheet's local vorticity thickness there, then the sheets are not isolated and flame shortening can occur. In addition to flame shortening another possibility is experimental error associated with the aqueous measurements. $P$ is estimated to be of the order of $500 \mu \mathrm{m}$ at low Reynolds number. Since the mixing is so slight, modest absolute inaccuracies yield large relative errors.

It is interesting to compare the results for a, shear layer with the work of Weddell (1941), who explored the mixing in a reacting, aqueous jet. A red solution of phenolphthalein and sodium hydroxide was injected into a tank of sulphuric acid solution from an axisymmetric nozzle. When the injected fluid mixed and reacted with the ambient solution, the red phenolphthalein became transparent and disappeared (the reverse reaction to the one employed in the present work). Weddell measured the visual length of the red jet from its origin to the station at which it disappeared and found that this reaction length became independent of jet Reynolds number above a Reynolds number of a few thousand. This result implies that the jet mixing is entrainment limited. Similarly in the present shear-layer results the mixing becomes entrainment limited when the three-dimensional, small-scale motions are established.

If the time-scale argument of Broadwell is correct (see §1) then some difference in the gaseous and aqueous mixing results for $R e \sim 10^{5}$ is expected. In the gaseous experiments, $T \equiv S c / R e^{\frac{1}{2}} \sim 10^{-2}$, while in the present aqueous work $T \sim 1$. Thus in the aqueous flow the entrainment time is comparable to the small-scale diffusion time so that the mixing rate may be reduced by delays from both processes. Un- 
fortunately, in order to match the gaseous value of $T \sim 10^{-2}$ in water, an aqueous Reynolds number of order $4 \times 10^{9}$ is required. Broadwell's argument predicts that at this Reynolds number the gas and water mixing rates should be identical. For $10^{5}<R e<10^{9}$ the aqueous mixing would be a very weak function of $R e$.

A somewhat different argument supposes that convection at the Kolmogorov microscale $\lambda_{0}$ contorts the reaction interface since the latter is the smaller of the two for $S c>1$. The interface thickness $\lambda_{i}$ is the Batchelor microscale (Batchelor 1959) and is of order $S c^{-\frac{1}{2}} \lambda_{0}$ (see $\S 3.1$ ). The ratio of Batchelor diffusion time to breakdown time is $\left(\lambda_{i}^{2} / \mathscr{D}\right) /(\delta / \Delta U) \sim S c^{-1} T \equiv R e^{-1}$ independent of Schmidt number. This question will be explored in a future paper.

\subsection{Effect of initial conditions}

Figure 13 also demonstrates the effect of freestream velocity ratio on the mixing transition. As $r=U_{2} / U_{1}$ is increased from $0 \cdot 38$ to $0 \cdot 76$, the transition Reynolds number $\Delta U \delta / \nu$ decreases. Thus the transition does not occur at a constant large-scale Reynolds number, implying that the three-dimensional transition is influenced by the initial conditions of the laminar vortex sheet.

Since the mixing transition is evidently influenced by the initial conditions of the shear layer, the normalized product thickness $P / \delta$ was plotted as a function of the initial Reynolds number $U_{1} \theta_{1} / \nu$ at a fixed $x$ for $0.38 \leqslant r \leqslant 0.80$ (figure 14). The curve in the figure represents thin-wake data $(r=1)$ and is discussed in $\$ 5.5$. This particular Reynolds number was chosen as the abscissa because (i) most of the vorticity in the initial vortex sheet originates in the boundary layer of the high-speed side when $r \equiv U_{2} / U_{1} \ll 1$, and (ii) most of the opposite-sign vorticity from the low-speed boundary layer is quickly cancelled by diffusion of the high-speed vorticity. When $r$ approaches unity, the two boundary layers are nearly symmetric so the choice of either one (or both) to characterize the initial conditions is clearly arbitrary. The initial vorticity thickness $\theta_{1}$ was estimated as

$$
\theta_{1}=\left(\nu \frac{x_{\mathrm{eff}}}{U}\right)^{\frac{1}{2}}
$$

where $x_{\text {eff }}$ is the effective origin of the laminar boundary layer in a zero pressure gradient. 'The value of $x_{\mathrm{eff}}$ was estimated to be $4 \mathrm{~cm}$ for this apparatus. There is considerable scatter in the data, especially at low Reynolds number and especially in the transition region, reflecting the sensitivity of the mixing to small-scale motions and the streamwise streaks in the transition. Even so, it is apparent that the transition occurs at about the same initial Reynolds number $L_{1}^{\prime} \theta_{1} / \nu$ for velocity ratios from $r=0.38$ to $r=0.80$. The result lends support to the conclusion that the mixing transition is determined by the initial conditions. The momentum thickness $\theta_{1}$ may not, of course, be the exact measure of the vortex-sheet thickness to define the transition Reynolds number, but it is a reasonable first approximation. Note that another parameter based on initial conditions $x / \theta_{1}$ varied with the flow speed. This point will be discussed in $\$ 6$.

\subsection{The three-dimensional instability}

There are at least three candidates for the initial instability which results in the wiggle. The Widnall instability (Widnall, Bliss \& Tsai 1974; Moore \& Saffman 1975; Tsai \& Willnall 1976: Saffman 1978) was first proposed to explain circumferential waves in 


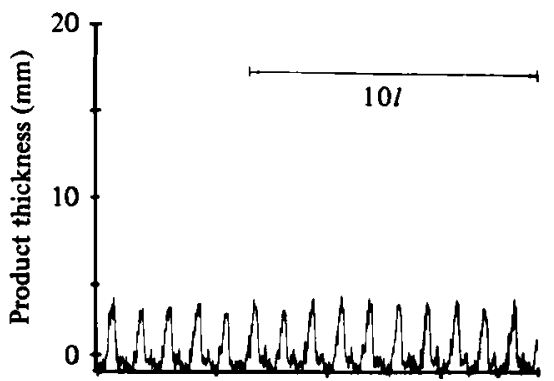

(a)

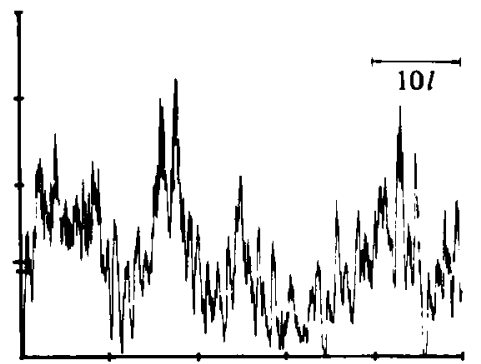

(c)

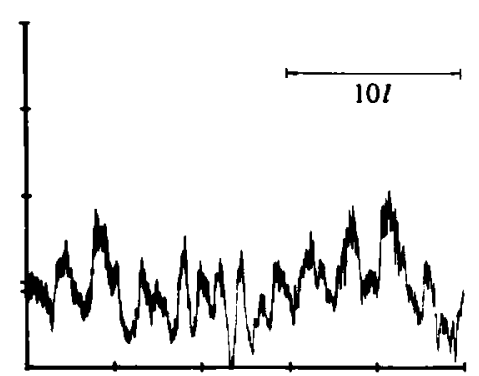

(b)

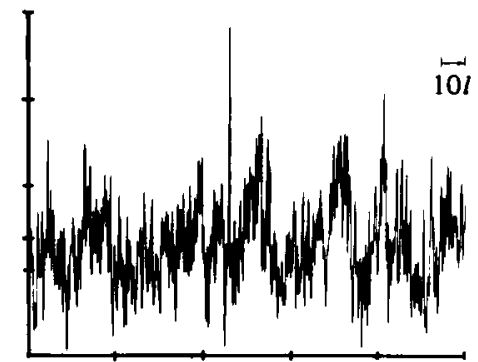

(d)

Fraune 15. Thick-wake time histories, $x=8.5 \mathrm{~cm}$. (a) $U d / \nu=220$; (b) $U d / \nu=2200 ;(c) U d / \nu=4200$; (d) $U d / \nu=15200$.

vortex rings and, as suggested by Saffman (private communication), may be responsible for the wiggle in the shear layer. Briefly, the physical idea is that, if a rectilinear line vortex is perturbed into a sinusoidal shape such that the vortex is steady under its own self-induced velocity, then in the presence of an external strain field the crests of the deformed line vortex are convected away from their unperturbed position as a consequence of the imposed strain. In this way the amplitude of the sinuous perturbation grows with time.

Benney (1961) and Corcos (1979) employed formal mathematical perturbation expansions to explore the infinitesimal three-dimensional instability. Brown (private communication) and Konrad (1977) have suggested that the Rayleigh-Taylor instability is responsible for the streamwise streaks. Their shadowgraph flow visualization did not display the wiggle, so their model does not directly incorporate it.

Further work is needed to determine which, if any, of these candidates is the initial three-dimensional instability in the shear layer. Of course, different instabilities may conceivably occur depending on the initial conditions and Reynolds number.

\subsection{Wake mixing}

The mixing in two different wake flows was briefly explored to compare with the shearlayer behaviour (see figure 1).

Figure 15 shows some typical time histories of the product thickness of the thick wake at $x=8.5 \mathrm{~cm}$ for four different wake Reynolds numbers $U d / \nu$. The signal was quite periodic for $U d / \nu=220$ and became less so as the Reynolds number increased. The average amount of product thickness, indicated on the vertical axis in each case, increased monotonically with $U d / \nu$ in the transition range, as shown in figure 16. The normalized product thickness $P / \delta_{w}$, is plotted as a function of the large-scale wake 


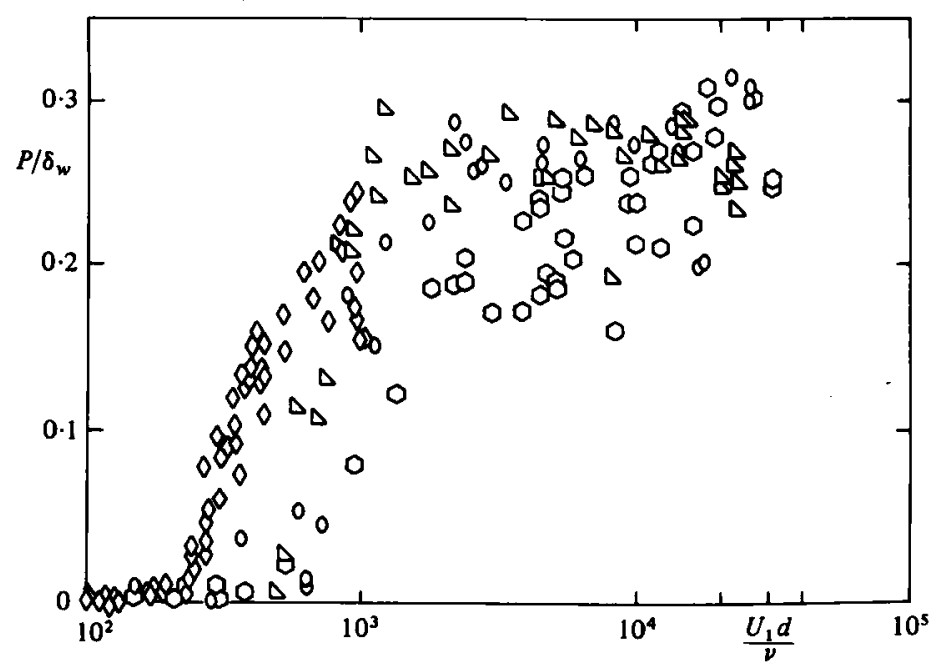

Figure 16. Effect of Reynolds number on wake mixing: $\diamond$, thin-plate wake, $x=16 \cdot 5 \mathrm{~cm}$, $d=2 \theta_{1} ; \bigcirc$, thick-plate wake, $x=8.5 \mathrm{~cm} ; \bigcirc$, thick-plate wake, $x=16 \mathrm{~cm}$; L, thick-plate wake, $x=32 \mathrm{~cm}$.

Reynolds number $U d / \nu$. The wake thickness $\delta_{w}$ is defined as $\delta_{w} \equiv(x d)^{\frac{1}{2}}$ and only approximately corresponds to the actual layer thickness since the expression is an asymptotic one and $x / d \sim 10$ here. No attempt was made to determine the precise virtual origin of the wake flow from mean velocity profiles. For the thin wake, $d$ is taken to be $2 \theta_{1}$. Both the thick and thin wakes exhibit a mixing transition across which the mixing jumps by an order of magnitude or more. Although there is considerable scatter in the data, it appears that the thick-wake mixing has not reached the asymptotic value at $x=8.5 \mathrm{~cm}$. The scatter for the thick-plate wake is larger than might be expected from experimental error, and may reflect spanwise variations in the mixing which are modulated at such a low frequency that the experiment averaging times were insufficient to produce a repeatable value.

A direct comparison of the thin wake and shear layer is presented in figure 14. The curved line represents the thin-wake data of figure 16. The two flows exhibit a transition in the mixing at the same initial boundary-layer Reynolds number $U_{1} \theta_{1} / \nu$. Since the definitions of $\delta$ and $\delta_{w}$ (equations (2.1) and (5.3), respectively) are arbitrary, there is no reason to expect the asymptotic values of $P / \delta$ and $P / \delta_{w}$ to be precisely equal, although they should be of the same order if $\delta$ and $\delta_{w}$ are logically defined. The remarkable similarity between the mixing transition in the two flows strongly suggests that the transition is determined by the characteristics of the initial boundary-layer vortex sheet and is only a weak function of velocity ratio.

\section{Discussion}

Two initial conditions which may influence the mixing transition are an initial Reynolds number and scale. The data of figure 14 were obtained at essentially a constant value of $x$. The measurement station was limited in the upstream direction because the shear layer and wake become very thin as $x$ is decreased. The relative measurement accuracy for $P$ and $\delta$ is poor noar the splitter plate. On the other hand, 


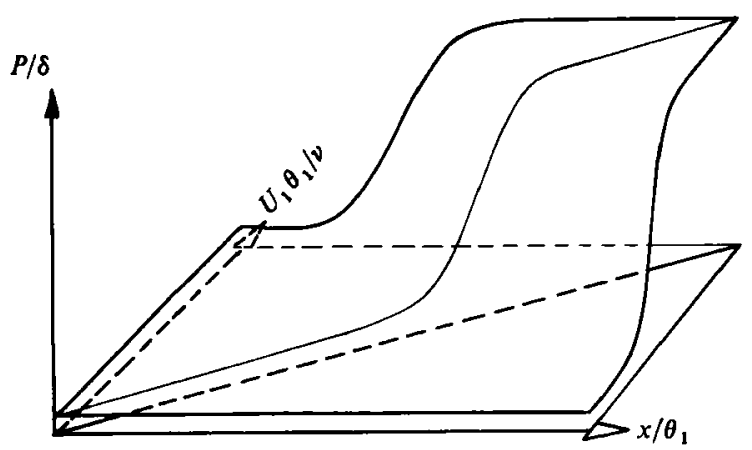

(a)

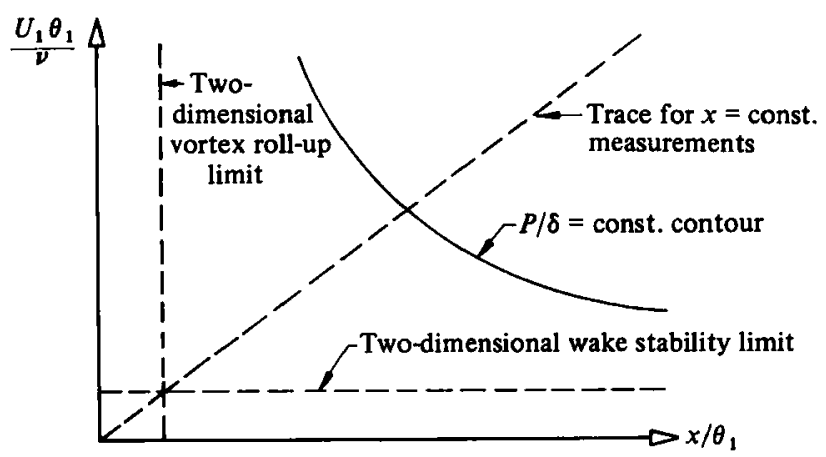

(b)

Fraure 17. Speculative effect of initial conditions on the mixing transition.

the finite size of the test section limits how far downstream reliable measurements can be made. Thus $x$ was not varied appreciably, even though the initial vortex-sheet scale $\theta_{1}$ varied with flow speed; thus the ratio $x / \theta_{1}$ was not held constant as the Reynolds number changed.

Bradshaw (1966) found that the approach to fully developed turbulence in the shear layer at the edge of a round jet $\left(r=U_{2} / U_{1}=0\right)$ correlated well with the parameter $U_{1} x / \nu$. Interestingly, $U_{1} x / \nu$ is just the product of $U_{1} \theta_{1} / \nu$ and $x / \theta_{1}$, a hyperbola in the $\left(U_{1} \theta_{1} / \nu\right),\left(x / \theta_{1}\right)$ plane. Based on this is the speculative relationship between the mixing and the initial conditions shown in figure 17. For small values of initial Reynolds number and near the splitter plate the mixing is slight since the flow (and the reaction interface) is two-dimensional. On the other hand, at large initial Reynolds number and far downstream the flow should contain three-dimensional small-scale motions and thus the mixing is entrainment limited there. Very near the splitter plate $\left(x / \theta_{1} \rightarrow 0\right)$ the mixing will be slight no matter how large $U_{1} \theta_{1} / \nu$, since the development of the three-dimensional motions almost certainly requires that the two-dimensional vortex sheet first roll up (a largely inviscid process which scales with the initial thickness). Far downstream the flow should be fully turbulent no matter how small the initial Reynolds number if $r \neq 1$. Thus the shear-layer transition curve should asymptote to $U_{1} \theta_{1} / \nu \rightarrow 0$ as $x / \theta_{1} \rightarrow \infty$. For $r=1$, however, the thin wake will not agree with the 
shear layer in the region of small $U_{1} \theta_{1} / \nu$ and large $x / \theta_{1}$ in figure 17. The wake transition curve should asymptote to a finite value of $U_{1} \theta_{1} / \nu$, in contrast to the shear layer.

The present measurements at constant $x$ trace out a diagonal path in the $\left(U_{1} \theta_{1} / \nu\right)$ $\left(x / \theta_{1}\right)$ plane of figure 17. Both $U_{1} \theta_{1} / \nu$ and $x / \theta_{1}$ vary as $U_{1}^{+}$. Figure 14 should therefore be viewed as a diagonal slice in figure $17(a)$, where both $U_{1} \theta_{1} / \nu$ and $x / \theta_{1}$ are varying. The slope of the diagonal trace $\left(U_{1} \theta_{1} / v\right) /\left(x / \theta_{1}\right) \sim x_{\mathrm{ett}} / x$ depends on the geometry of the apparatus $\left(x_{\mathrm{etf}}\right)$ and the measurement location $x$. The slope of the transition contour in figure $17(b)$ steepens with increasing initial Reynolds number so that $x / \theta_{1}$ at transition does not vary much for large $U_{1} \theta_{1} / \nu$. As a consequence, it is possible to roughly categorize transition behaviour at large initial Reynolds number of the basis of only one parameter, $x / \theta_{1}$.

The aqueous mixing transition is complete at $x / \theta_{1} \doteq 500$. Bradshaw (1966) found that the time-averaged quantities $\overline{u^{\prime}}, \overline{v^{\prime}}$ and $\overline{u^{\prime} v^{\prime}}$ all approach their asymptotic levels at $x / \theta_{1}=1000$, in approximate agreement with the end of the mixing transition. Jimenez, Martinez-Val \& Rebollo (1979) measured power spectra of the velocity fluctuations in a shear layer. They report that the character of the spectrum in the inertial range changed from a steep slope that may be -3 to a slope of about $-\frac{5}{3}$ and that this spectrum transition occurs at the same Reynolds number as the mixing transition. Interestingly, Jimenez et al. point out that an exponent of -3 is expected in two-dimensional turbulence (Batchelor 1969). Thus the mixing transition resulting from the development of three-dimensional, small-scale motions is detectable in both time-averaged and spectral measurements of the velocity field and apparently corresponds to the approach to an asymptotic turbulent state.

Finally, it is worth while to note from a modelling standpoint that the mixing is not directly correlated with Reynolds stress. This is illustrated by comparison with the measurements of Bradshaw (1966) who found a maximum in $\overline{u^{\prime} v^{\prime}}$ in the transition region. He reported that $\left(\overline{u^{\prime} v^{\prime}}\right)_{\max }$ considerably overshoots its final, asymptotic value for 'fully developed turbulence', then rapidly declines to a local minimum (which may be even slightly below the asymptotic value), increases to a second local maximum less than the first, and finally decreases monotonically to the asymptotic value. Tripping the boundary layer eliminates these undulations and $\left(\overline{u^{\prime} v^{\prime}}\right)_{\max }$ very slowly approaches the final level monotonically from below. Bradshaw noted that the first Reynolds stress peak apparently corresponds to vortex pairing ("confluence of vortex rings'); however, he attributes the second peak to 'the establishment of the shearproducing part of the turbulence spectrum; the shear stress is higher than the fully developed value because the smaller-scale turbulence which drains energy from the shear-producing eddies by a sort of "eddy-viscosity" mechanism, has not yet been established'. An alternative explanation is that both peaks are a result of vortex pairing. The first peak in $\overline{u^{\prime} v^{\prime}}$ is very narrow and high because there is relatively little jitter in the streamwise location of the first pairing. The second peak is lower and wider than the first because the jitter in pairing location increases with $x$. Co-operative, large-scale transport of momentum by the vortices is then responsible for the high correlation in velocity fluctuations, $\overline{u^{\prime} v^{\prime}}$. In contrast, the mixing is sensitive to the presence of the small scales and is not directly connected with the Reynolds stress. Further evidence is from Wygnanski, Oster \& Fiedler (1979), who observed negative values of Reynolds stress in a forced shear layer. The molecular-scale mixing rate, on the other hand, is always positive. 


\section{Conclusions}

In summary the effect of Schmidt number on the molecular-scale mixing at low Reynolds number is pronounced, while at high Reynolds number the mixing changes by only a factor of 2 or less for a three orders of magnitude variation in Schmidt number. Above the transition, the mixing is essentially independent of $R e$. This is consistent with qualitative predictions of Broadwell's mixing model. A transition region for the mixing, first observed by Konrad in a gas flow, was again found to correspond to the introduction of small-scale three-dimensional motions into the layer. The aqueous mixing is very sensitive to the presence of these small scales. The large-structure transition Reynolds number was found to vary with velocity ratio, implying that the instability is not a unique function of the large-scale Reynolds number $\Delta U \delta / \nu$ as would be the case for the temporal problem of a vortex sheet which is infinitely thin at $t=0$. The initial conditions of the finite, laminar vortex sheet leaving the splitter plate are believed to be important in determining the transition. The velocity ratio appears to have relatively small influence on the transition compared to the initial scale and Reynolds number of the higher-speed boundary layer. Further work is necessary to determine the precise shape of the transition curve and its dependence on velocity ratio, freestream turbulence, initial vortex-sheet perturbations, spanwise location and other parameters.

The unique flow visualization provided by the chemistry revealed a spanwisesinuous ' wiggle' which is believed to play an important role in introducing streamwise vortices and small-scale motions in the mixing layer. The initial instability which generates the wiggle is not yet known, although several oandidates exist. The precise evolution of the wiggle and its effect on spanwise variations of the mixing in the mixing layer remain uncertain.

The global structure of other turbulent flows may be fruitfully studied in the future using the reacting flow-visualization technique. The technique complements conventional methods using inert tracers since it emphasizes regions of intense mixing. To the extent that these regions correspond to rotational fluid, the reaction product displays the global vorticity.

The author is indebted to Professor Anatol Roshko for his guidance and advice during the course of these experiments. The ideas presented here were developed during discussions with the faculty and students of the Graduate Aeronautical Laboratory (GALCIT). Most of the wake measurements were done with the help of Till W. Liepmann. The author also wishes to acknowledge the significant contributions of James E. Broadwell, Garry L. Brown, and Anatol Roshko in reviewing the manuscript. The work was made possible by the Air Force Office of Scientific Research under contract F44620-76-C-0046. 


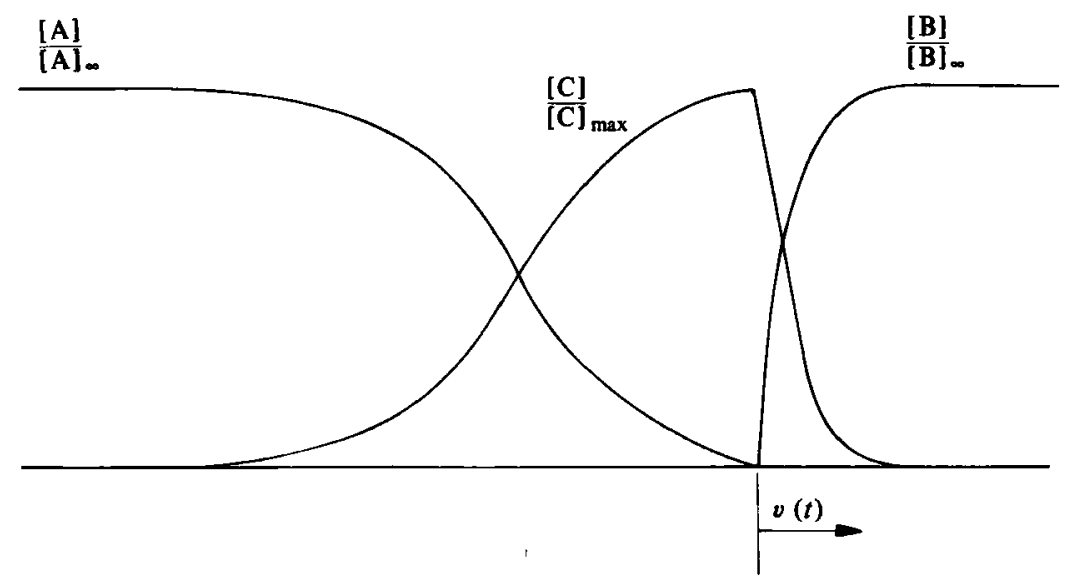

Figure 18. Concentration profiles across a plane reacting interface. The reaction surface moves to the right with speed $v(t)$.

\section{Appendix}

Phenolphthalein is a $\mathrm{pH}$ indicator with a transition interval from about $\mathrm{pH}=8$ (clear) to $\mathrm{pH}=10.5$ (red). Half of the phenolphthalein is converted to the red form at $\mathrm{pH}$ of 9.3. In order to approximate an irreversible chemical reaction, it is necessary to minimize the disappearance of the red product due to the reverse reaction. This was done by using an excess of $\mathrm{OH}^{-}$in the alkaline stream. The temporal problem of a plane reaction interface is sketched below.

Consider for the moment the diffusion-limited irreversible reaction

$$
\mathrm{A}+\mathrm{B} \longrightarrow \mathrm{C} \text {. }
$$

At $t=0$ a membrane disappears separating two half-spaces, the left one containing a uniform concentration of $A$ and the right one uniform with reactant $B$. For $t>0$, the concentration profiles of the reactants and product are error functions. If the equivalence ratio $f \equiv[\mathrm{A}]_{\infty} /[\mathrm{B}]_{\infty}$ is unity, then the reaction surface is stationary. In the case of an excess of reactant A, however, Marble \& Broadwell (1977) show that the surface moves to the right (see figure 18) with respect to the fluid at a speed

$$
v(t)=\alpha\left(\frac{\mathscr{D}}{t}\right)^{\frac{1}{2}}, \quad \text { where } \quad \operatorname{erf} \alpha=\frac{f-1}{f+1} .
$$

For $f \gtrsim 10, v(t) \simeq(\mathscr{D} / t)^{\frac{1}{2}}$. Product is formed at the reaction surface and tends to be left behind in a region of increasing reactant $A$ as the surface moves to the right.

In the phenolphthalein system we can force the bulk of the red product to be left behind in a region of increasing $\mathrm{pH}$ by using an excess of $\mathrm{OH}^{-}$reactant. The red product will thus tend to remain red and not revert back into the colourless reactants. In practice the amount of excess $\mathrm{OH}^{-}$reactant is limited by a separate reaction which causes the red form to disappear for $\mathrm{pH}>12$. A pH value of about 11.7 was selected as a compromise. 


\section{REFERENCES}

Batchelor, G. K. 1959 Small scale variation of convected quantities like temperature in a turbulent fluid. Part I. J. Fluid Mech. 5, 113.

BAtchelor, G. K. 1969 Computation of the energy spectrum in homogeneous 2-D turbulence. Phys. Fluids Suppl. 12, II 233.

Bernal, L. P., Breidenthal, R. E., Brown, G. L., Konrad, J. H. \& Roshko, A. 1979 On the development of three-dimensional small scales in turbulent mixing layers. 2 nd Symp. on Turbulent Shear Flows, Imperial College, London.

Benney, D. J. 1961 A non-linear theory for oscillations in a parallel flow. J. Fluid Mech. 10, 209.

Bradshaw, P. 1966 The effect of initial conditions on the development of a free shear layer. J. Fluid Mech. 26, 225.

BreidenthaL, R. E. 1979 Chemically reacting, turbulent shear layer, A.I.A.A. J. 17, 310-311. (Also Ph.D. thesis, California Institute of Technology, 1978.)

Brown, G. L. \& Roshкo, A. 1974 On density effects and large structure in turbulent mixing layers. J. Fluid Mech. 64, 775.

Caldin, E. F. 1964 Fast Reactions in Solution, pp. 66-67. Wiley.

Concos, G. M. 1979 The mixing layer : deterministic models of a turbulent flow. Univ. Califormia, Berkeley, Rep. FM-79-2.

Czerlinski, G. \& Eigen, M. 1959 Eine Temperaturesprungmethode zur Untersuchung chemischer Relaxation. Z. Electrochemie 63 (6), 652.

Dimotakis, P. E. \& Brown, G. L. 1976 The mixing layer at high Reynolds number: largestructure dynamics and entrainment. J. Fluid Mech. 78, 535.

Jimenez, J., Martinez-VAL, R. \& Rebollo, M. 1979 The spectrum of large-scale structures in a mixing layer. 2nd Symp. on Turbulent Shear Flows, Imperial College, London, p. 8.7.

Koltorf, I. M. 1937 Acid-Base Indicators, p. 221. Macmillan.

Konread, J.H. 1977 An experimental investigation of mixing in two-dimensional turbulent shear flows with applications to diffusion-limited chemical reactions. Ph.D. thesis, California Institute of Technology. (Also Project SQUID Tech. Rep. CIT-8-PU, December 1976.)

Marble, F. E. \& Broadwell, J. E. 1977 The coherent flame model for turbulent chemical reactions. TRW Rep. 29314-6001-RE-00.

Moore, D. W. \& Saffman, P. G. 1975 The instability of a straight vortex filament in a strain field. Proc. Roy. Soc. A 346, 413.

Roshko, A. 1976 Structure and turbulent shear flows: a new look. A.I.A.A. J. 76-78, Dryden Research Lecture. (Also A.I.A.A. J. 14, 1349-1357).

Safrman, P. G. 1978 The number of waves on unstable vortex rings. J. Fluid Mech. 84, 625.

Toor, H. L. 1962 Mass transfer in dilute turbulent and non-turbulent systems with rapid irreversible reactions and equal diffusivities, A.I.Ch.E. Journal 8, no. 1, 70.

Tsai, C.-Y. \& Widnald, S. E. 1976 The stability of short waves on a straight vortex filament iu a weak externally imposed strain field, J. Fluid Mech. 73, 721.

Weddell, D. 1941 Turbulent mixing in gas flames, p. 115. Ph.D. thesis, Massachusetts Institute of Technology.

Widnall, S. E., Bliss, D. B. \& Tsai, C.-Y. 1974 The instability of short waves on a vortex ring. J. Fluid Mech. 66, 35.

WinaNT, C. D. \& Browand, F. K. 1974 Vortex pairing: the mechanism of turbulent mixing layer growth at moderate Reynolds number. J. Fluid Mech. 63, 237.

Witte, A. B., Broadweld, J. E., Shackleford, W. L., Cummings, J. C., Trost, J. E., Whiteman, A. S., Marble, F. E., Crawford, D. R. \& Jacobs, T. A. 1974 Aerodynamic reactive flow studies of the $\mathrm{H}_{2} \mathrm{~F}_{2}$ laser-II. Air Force Weapons Lab., Kirtland Air Force Base, New Mexico, AFWL-TE-74-78, 33.

Wygnanski, I., Oster, D. \& Fiedler, H. 1979 The forced, plane, turbulent mixing layer: a challenge for the predictor. 2nd Symp. on Turbulent Shear Flows, Imperial College, London, p. 8.12. 